\title{
Three-dimensional simulation of asphalt mixture incorporating aggregate size and morphology distribution based on contact dynamics method
}

\author{
Haitao Ge ${ }^{\text {a, }}$, Juan Carlos Quezada ${ }^{\text {a }}$, Vincent Le Houerou ${ }^{\mathrm{b}}$, Cyrille Chazallon ${ }^{\mathrm{a}}$ \\ ${ }^{\text {a }}$ Université de Strasbourg, INSA de Strasbourg, CNRS, ICube, UMR 7357, F-67000 Strasbourg, France \\ ${ }^{\mathrm{b}}$ Université de Strasbourg, CNRS, ICube, UMR 7357, F-67000 Strasbourg, France
}

\section{A R T I C L E I N F O}

\section{Keywords:}

Morphology characterization

Contact dynamics method

3D laser scanning

Particle tessellation

Complex modulus

Discrete element modelling

\begin{abstract}
A B S T R A C T
Aggregate size and morphology distribution have crucial influences on the performance of asphalt mixtures. In recent decades, the discrete element method (DEM) has been widely adopted to reconstruct aggregate particles and simulate the performance tests of asphalt mixtures. However, most simulations assume a simplification in the particle shape and neglect irregular particle features and its morphology distribution. In this study, based on the acquisition of three-dimensional (3D) aggregate shapes using laser scanning, the morphological characteristics for a large number of particles were analyzed statistically by image processing technology. Numerical asphalt mixture samples were generated by Voronoi tessellations combining the particle size distribution and morphology statistics. The model reliability has been validated by conducting experiments and simulations based on the Contact Dynamics (CD) method for the complex modulus test. Using this procedure, the aggregate morphology and size distribution could be incorporated in numerical simulations of asphalt mixtures. It also implies a significant computational-time reduction by skipping several preparation stages for polyhedral samples. The simulation method can be used to study granular systems as inclusion in a solid matrix such as asphalt mixtures. Furthermore, the micro-mechanism of mixture damage initiation can be revealed at the particle-scale by using the proposed method.
\end{abstract}

\section{Introduction}

Asphalt mixture is one of the main materials for wearing courses and base layers for high traffic pavements. The mechanical behavior of this composite material composed of aggregates, bitumen and air depends highly on the properties of its components in interaction [1]. Experiments are generally used to determine viscoelastic material properties from a macro-scale. However, it is still a challenge to study the micromechanical behavior of the material through laboratory measurements. With the continuous advances in computation capacity, numerical methods are widely used for examining micro-mechanically material performances. The discrete element method (DEM) has been demonstrated to be an effective method to simulate granular systems including asphalt mixtures at the particle-scale over the last decades [2-5].

For a simulation of asphalt mixture, it is required to generate the mesoscopic structure of the mix. As the geometries of aggregate particles play an essential role in the mixture performance [6-10], establishing a numerical model close to the real particle assembly is an issue that deserves much attention. Image technique was widely adopted to characterize the internal structure of asphalt mixture for numerical simulation in the two-dimensional (2D) $[11,12,5,13]$ and threedimensional (3D) DEM models [14-16]. However, image-based models are dependent on the prepared physical specimens; it is not capable of providing an absolute virtual environment for numerical simulations of asphalt mixtures. Sensitivity analysis of component properties such as aggregate morphology and spatial distribution based on this method is also limited due to the difficulty of controlling these factors precisely in the sample preparation.

Regarding the statistical assessment of the variation for mixture composition, the structure reconstruction based on image-based methods is limited [17]. Consequently, establishing a strategy to flexibly generate numerical samples resembling real asphalt specimens is suitable for creating a fully virtual environment and estimating systematically effective properties for asphalt mixtures.

Currently, some researches were conducted on the mixture simulation composed of random particle shapes, based on computer-aided algorithms [18-22] and image scanning devices [23-25]. However, in

\footnotetext{
* Corresponding author.

E-mail address: haitao.ge@insa-strasbourg.fr (H. Ge).
} 
most of these studies, the particles were modeled as simplified clumps composed of limited overlapping spheres or disks [26], neglecting crucial particle features such as angularity. This simplification may affect granular fabric properties such as porosity, contact anisotropy, and force chains network $[27,28]$. In addition, although high-resolution particle clumps can properly simulate real particles, the huge computation cost is not affordable yet. On the other hand, current numerical models only treat the aggregate size distribution explicitly, particle shapes used in the current research are usually created or selected randomly in the simulation and do not consider the aggregate morphology distribution in the real assembly. Moreover, the preparation of numerical samples composed of polyhedral particles is an extraordinarily time-consuming process: complex algorithms are required in contact detection and repulsive force computation during the preparation stages, such as gravity deposition, compaction, and stabilization of numerical samples.

In this study, based on the acquisition of aggregate shapes through 3D laser scanning, the morphological characteristics for a large number of real particles were analyzed statistically by using image processing technology. The contact dynamics (CD) method was introduced to simulate irregular particles because of its suitability for polyhedron modeling. The numerical asphalt mixture specimens were generated by a tessellation method considering both the aggregate size distribution and morphology data. The numerical and experimental complex modulus tests were carried out with different temperatures and loading frequencies to confirm the model reliability. Finally, the micromechanical analysis was conducted to investigate the contact force distribution between particles and reveal the essence of mixture damage initiation at the particle-scale. The simulation method proposed in this study incorporate aggregate size and morphology distribution for asphalt mixture modeling, it could provide a basis for establishing highprecision material models and optimal pavement design.

\section{Morphology distribution of aggregate particles}

\subsection{Three-dimensional laser scanning of aggregate particles}

In this study, aggregate particles with sieving sizes from $4 \mathrm{~mm}$ to $31.5 \mathrm{~mm}$ were randomly selected from the stockyard. A threedimensional laser scanner (FARO Edge ScanArm HD) was used to obtain real geometrical data of the selected 500 aggregates. Surface point coordinates of particles were collected and transmitted to the computer during the scanning process. After the scanning and data acquisition of all specimens, the point cloud data obtained by the laser scanner were imported into MATLAB to further process individually, as Fig. 1 shows.

\subsection{Statistical analysis for aggregate form}

The traditional method usually divides particle morphology into three scales: contour form, angularity, and surface texture [29], among which contour form and angularity belong to the macroscopic and mesoscopic scale, respectively, while surface texture belongs to the microscopic scale. Shape characteristics of particles with different scales affect various aspects of the mixture performance [30-32]. In the numerical particle-based simulation, the surface texture of particles can be indirectly implemented by setting a friction coefficient of mutual interaction. In the conventional simulation, the friction coefficient is usually set to a constant value $[33,34]$. Accordingly, in this study, particle surface texture statistics has not been carried out.

The aggregate form is used to describe the dimension characteristics of a particle, defined by the ratios of three linear dimensions: length (L), width (I), and thickness (S). Elongation ratio $(\mathrm{LI}=\mathrm{L} / \mathrm{I})$ and Flatness ratio (IS $=\mathrm{I} / \mathrm{S}$ ) evaluate how a particle resembles a column or a plate [35]. If the value of LI and IS is close to 1, the aggregate is not slender. Otherwise, the particle is elongated or flatted. The minimum bounding box algorithm [36] was performed to calculate the minimum bounding box for a particle so that the dimension values of L, I, and S can be extracted. Schematic drawings of one aggregate in different bounding boxes are illustrated in Fig. 2.

Statistical features of LI and IS of all particles were analyzed. Fig. 3a and $\mathrm{b}$ show the probability distribution histogram of the LI and IS, respectively. As shown in the figures, both LI and IS of aggregate particles obey a normal distribution. Aggregates with IS less than 0.6 are defined as flaky particles and constitute about $5 \%$ of the overall samples, which fulfills the French standard stating flaky aggregates used in road construction should be less than 15\% (NF P18-545 [37]).

\subsection{Statistical analysis for aggregate angularity}

Angularity belongs to mesoscopic shape characteristics. If particles show sharp edges, it will be more favorable to the mixture interlocking. If aggregates are rounded, the overall mixture strength will be significantly reduced, even with better gradation. The Sphericity value $(S p=$ $\left.\sqrt[3]{36 \pi V^{2}} / A\right), \mathrm{V}$ and $\mathrm{A}$ represent particle volume and surface area, respectively, witnesses the angularity of aggregate particles [38,39]. The closer this value to 1 , the more spherical a particle, and the lower the particle angularity.

The statistics of $\mathrm{Sp}$ were analyzed using the same method as mentioned above, as Fig. 4 reports. The results show that the Sp obeys quite well a normal distribution. Table 1 gives the statistical results of aggregate morphology. The average aspect ratio (L: I: S) for aggregates is about 1.815: 1.301: 1 , the mean and standard deviation of the Sp are 0.8013 and 0.0428 respectively.

\section{Complex modulus test}

\subsection{Experimental set-up}

To identify the mechanical properties of asphalt mixtures, complex modulus tests were conducted in a two-point bending (2 PB) configuration according to the EN 12697-26:2012 specifications [40], the test configuration is shown in Fig. 5.

The slab specimens were prepared according to the asphalt mixture gradation (Fig. 6) and the trapezoidal samples were cut from the slab according to the dimensions given in Table 2. In the 2 PB tests, the loading frequencies ranged from $3 \mathrm{~Hz}$ up to $40 \mathrm{~Hz}$, and temperatures varied between $-10{ }^{\circ} \mathrm{C}$ and $30{ }^{\circ} \mathrm{C}$. For each couple temperaturefrequency, a sinusoidal horizontal displacement $Z=A \sin (\omega t)$ was measured on the top of each sample and the amplitude A was $63 \mu \mathrm{m}$

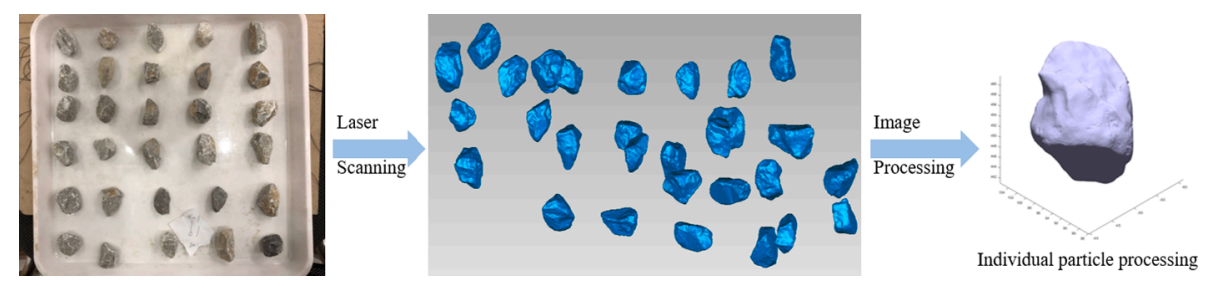

Fig. 1. 3D scanning process. 

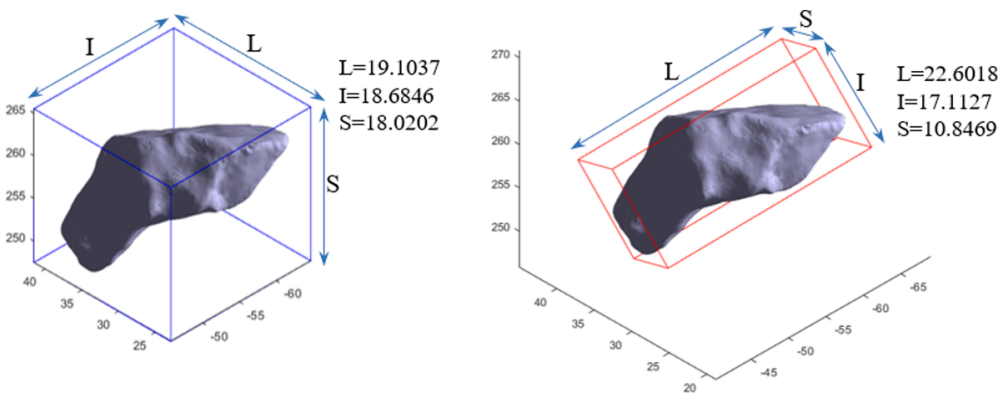

Fig. 2. Original bounding box of an aggregate particle (left) and minimum bounding box cube of the aggregate particle (right).

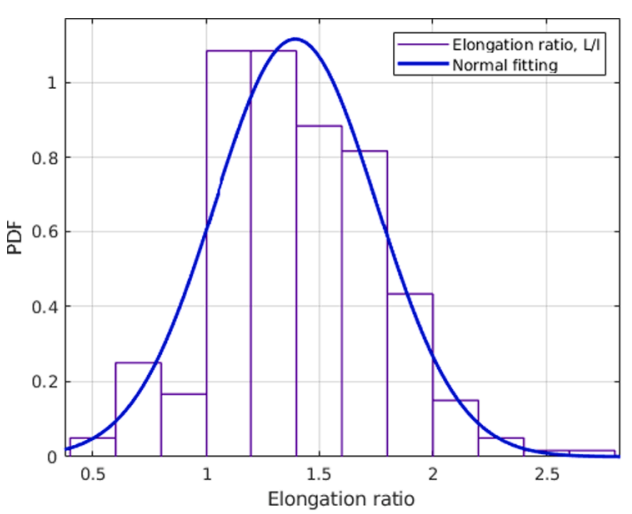

(a)

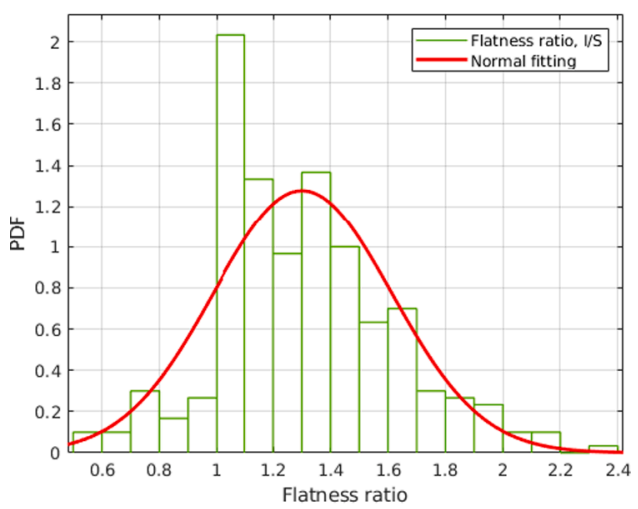

(b)

Fig. 3. Probability distribution and normal fitting: (a) Elongation ratio (LI); (b) Flatness ratio (IS).

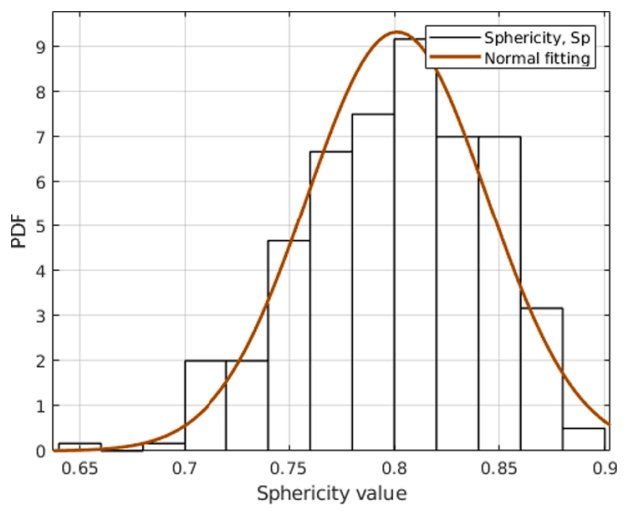

Fig. 4. Probability distribution and normal fitting of Sphericity (Sp).
Table 1

Statistical parameters for aggregate morphology.

\begin{tabular}{llll}
\hline Parameters & Distribution type & Mean & Standard deviation \\
\hline LI & Normal & 1.3951 & 0.3578 \\
IS & Normal & 1.3009 & 0.3124 \\
Sp & Normal & 0.8013 & 0.0428 \\
\hline
\end{tabular}

leading to a maximum strain $\in$ less than $50 \times 10^{-6}$ to prevent fatigue damage.

\subsection{Laboratory results}

The viscoelastic behavior of an asphalt mixture under cyclic loading can be assessed by the dynamic modulus $\left|E^{*}\right|$ and the phase angle $\Phi$. To determine these viscoelastic parameters, the real part $E_{1}$ and the imaginary part $E_{2}$ of the dynamic modulus must be calculated for each couple temperature-frequency. These values are provided as following [40]:

$\left|E^{*}\right|=\sqrt{E_{1}^{2}+E_{2}^{2}}$

$\Phi=\arctan \left(E_{1} / E_{2}\right)$

$E_{1}=\gamma\left(\frac{F_{0}}{z_{0}} \cos \psi+10^{-6} \mu \omega^{2}\right)$

$E_{2}=\gamma \frac{F_{0}}{z_{0}} \sin (\psi)$

$\gamma=\frac{12 h^{3}}{e(B-b)^{3}}\left[\left(2-\frac{b}{2 B}\right) \frac{b}{B}-\frac{2}{3}-\ln \frac{b}{B}\right]$

$\mu=0.135 M+m$

Where:

- $F_{0}, z_{0}$ and $\psi$ are the peak values of force, displacement and initial phase angle measured during the last ten seconds of each test, respectively;

- $\gamma\left(\mathrm{mm}^{-1}\right)$ is the form factor depending on the geometrical values;

- $\mu=0.135 M+m$ is the mass factor influencing the resultant force by inertial effects. In this study, the mass of each trapezoidal sample $\mathrm{M}$ is in average $0.6 \mathrm{~kg}$ and the mobile equipment mass at the head of the specimen $\mathrm{m}$ is $0.235 \mathrm{~kg}$.

The dynamic modulus $\left|E^{*}\right|$ and phase angle $\Phi$ were measured at six temperatures $\left(-10,0,10,15,20\right.$ and $\left.30^{\circ} \mathrm{C}\right)$ and five loading frequencies $(3,6,10,25$, and $40 \mathrm{~Hz})$, the results are shown in Fig. 7 . 

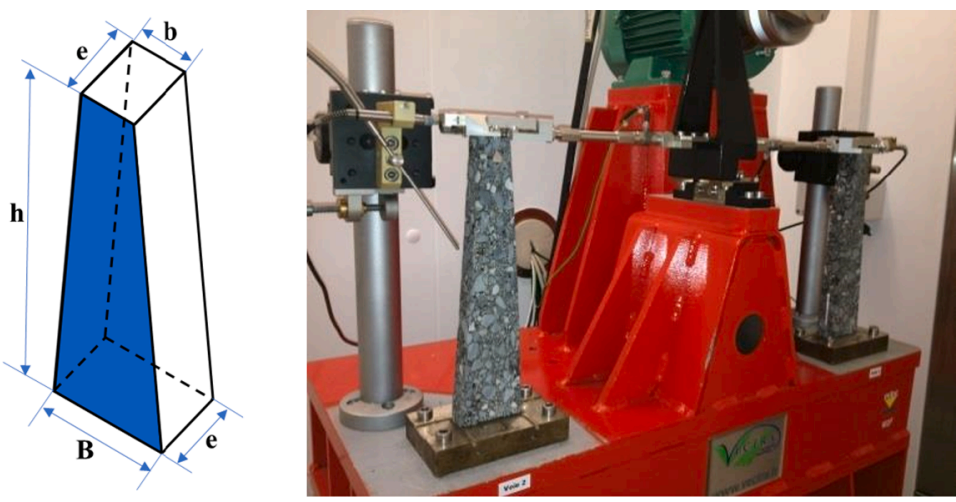

Fig. 5. Trapezoidal specimen and 2 PB test process [41].

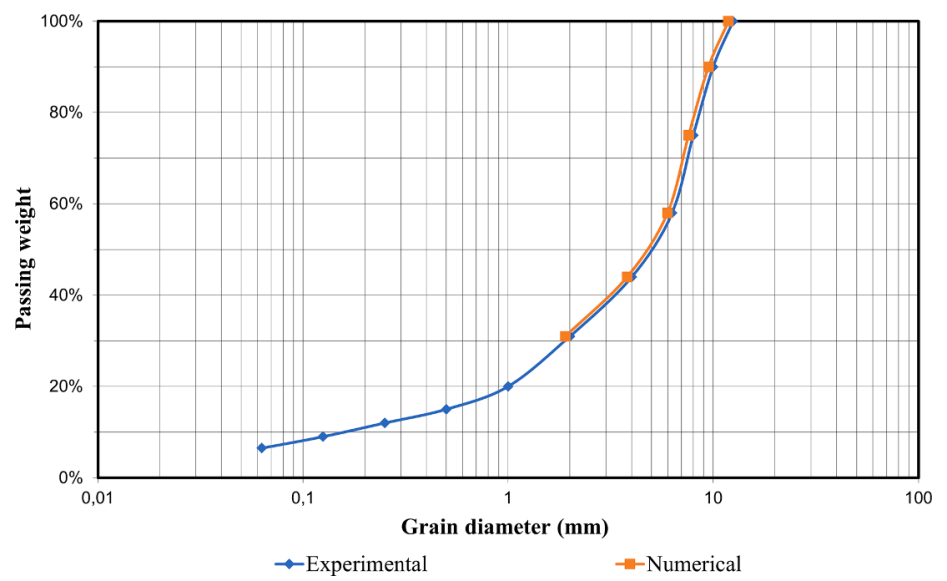

Fig. 6. Particle size distribution (PSD) of asphalt mixture.

Table 2

Dimensions of the specimens: EN 12697-26:2012.

\begin{tabular}{ll}
\hline $\begin{array}{l}\text { Dimensions of the } \\
\text { specimens }\end{array}$ & $\begin{array}{l}\text { Type of mixture } \\
D \leq 14 \mathrm{~mm}\end{array}$ \\
\hline B & $56 \pm 1 \mathrm{~mm}$ \\
$\mathrm{~b}$ & $25 \pm 1 \mathrm{~mm}$ \\
$\mathrm{e}$ & $25 \pm 1 \mathrm{~mm}$ \\
$\mathrm{~h}$ & $250 \pm 1 \mathrm{~mm}$ \\
\hline
\end{tabular}

Note: D is the maximum aggregate size.

\section{Numerical procedures}

\subsection{Contact dynamics method}

The discrete element method (DEM) aims to model the collective behavior of a media composed of distinct interacting particles. In computational contact mechanics, the contact problem is an extremely non-linear, non-smooth, and non-differential issue. In terms of contact condition calculation, DEM can be divided into two families of numerical methods: 1) "Smooth" (regular) explicit approaches, pioneered by P. Cundall $[42,43]$, assimilating grain interpenetration to possible deformation at the contact and 2) "Non-Smooth" (non-regular) implicit approaches, based on contact dynamics (CD) method, which take into account shocks without grain interpenetration [44-46]. Fig. 8 gives the comparison schemes of these two methods in solving the Signorini contact condition and the Coulomb's friction law.

In the $\mathrm{CD}$ method, the contact laws are non-differential steep laws defining the relation between relative velocities $\boldsymbol{U}$ and impulses $\boldsymbol{R}$. These laws are based on the Signorini condition and Coulomb's friction law for perfectly rigid bodies.

The contact laws are managed with an implicit method using a NonLinear Gauss-Seidel algorithm (NLGS) at each time step. All kinematic constraints implied by contacts between particles are computed simultaneously by combining with the equations of dynamics, in order to determine all the velocities and contact forces in the system. Furthermore, the implicit method enables us to correctly compute equilibrium states, which is not always the case with explicit methods [47].

A significant advantage of the $\mathrm{CD}$ method is that particle dynamics determine the characteristic system time so that a larger time step can be used compared to the explicit DEM method. Because of its advantages, we adopted the $\mathrm{CD}$ method for our simulation of asphalt mixture consisting of polyhedral aggregate particles. The open-source software LMGC90 ( https://git-xen.lmgc.univ-montp2.fr/lmgc90/1mgc90_user/ -/wikis/home) is adopted in which CD method is built-in.

\subsection{Irregular polyhedral particles simulation}

Asphalt mixtures are complex multi-phase systems composed of aggregates, bitumen and air, where aggregates occupy almost $95 \%$ of the mass in the mix. Consequently, asphalt mixture can be seen as a system of irregular particle tessellations from a geometrical point of view (Fig. 9), and particles interact with each other through a viscoelastic mastic phase containing bitumen and air between them $[17,39,48]$. Therefore, the open-source software NEPER [49,50] was adopted to generate particle tessellation samples. It offers support for Voronoi tessellations in both 2D and 3D, large tessellations can be easily created, and any convex domain shape can be used. Moreover, tessellations can also be written in various formats for different simulations. 


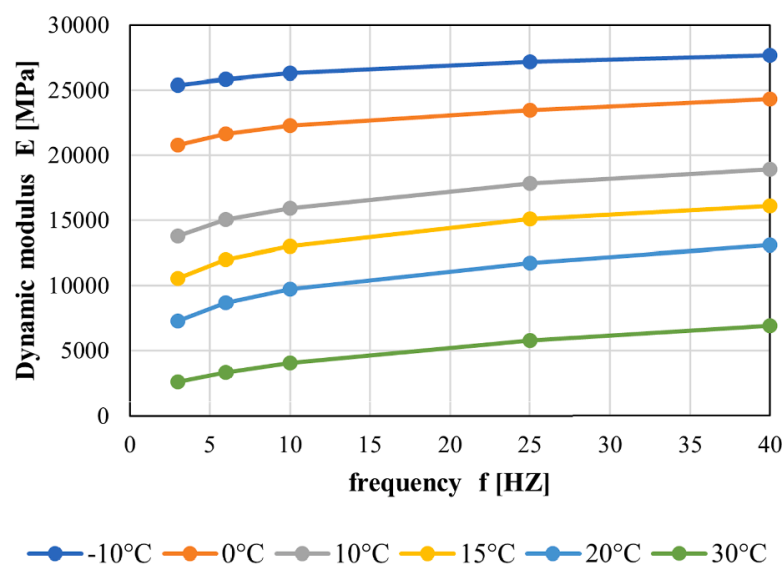

(a)

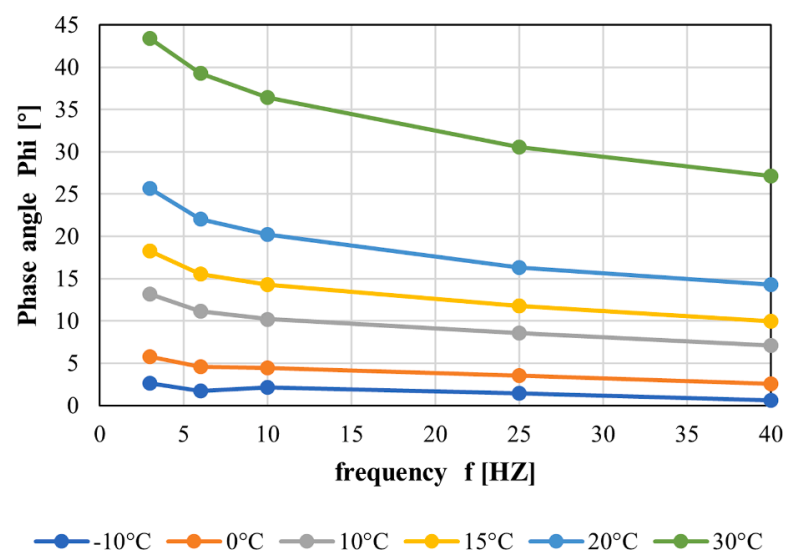

(b)

Fig. 7. Dynamic modulus and phase angles from experimental measurements of asphalt mixture: (a) Dynamic modulus; (b) Phase angle.

Before generating the trapezoidal numerical specimen for a 2 PB test, two main parameters need to be input. The first is particle size: we input the size of aggregates according to the PSD results (Fig. 6) to simulate aggregate gradation. The second is particle morphology: we input the aggregate shape parameters from the statistics reported in Table 1, including aspect ratio and sphericity and its distribution. Using this procedure, we can achieve the goal of incorporating the aggregate morphology and aggregate size in the numerical simulation of asphalt mixtures. After the generation, about 5,680 tessellations were created following the experimental gradation cut at $2 \mathrm{~mm}$ to reduce the total quantity of particles in the sample, so that finer aggregates are included in the asphalt mastic. For particles between $2 \mathrm{~mm}$ and $4 \mathrm{~mm}$, we assume that they have the same morphology distribution as other particles.
Then, the vertices of each tessellation were imported in LMGC90 to build numerical models. In this stage, particles were generated based on the convex hull of vertices from the tessellation. The initial generated tessellation is not proper for modeling the asphalt mixture because there are no gaps between grains and particles touch seamlessly. Therefore, the original particle size from net tessellation is shrunk by multiplying a correction factor $f_{c r}$ (Eq. (7) [48]) in order to create gaps accommodating the mastic phase among particles. As Fig. 10 shows, rigid particles interact with each other through the viscoelastic asphalt mastic.

$f_{c r}=\left(1-\frac{T_{L}}{100} \frac{\rho_{S}}{\rho_{L}}\right)^{\frac{1}{3}}$

Where:

- $T_{L}$ is the binder content (4.7\%), $\rho_{S}$ the initial density of particles $\left(2.6 \mathrm{Mg} / \mathrm{m}^{3}\right)$ and $\rho_{L}$ the density of binder $\left(1.03 \mathrm{Mg} / \mathrm{m}^{3}\right)$.

After the sample generation, the contact model was activated between particles. To simulate the mobile loading parts in the experimental device, a top plate was generated with mass of $0.235 \mathrm{~kg}$ and putted over the sample. Finally, the system was stabilized by activating gravity. Following this entire process, numerical samples were prepared with the same geometrical properties as those identified on experimental specimens. Figs. 6 and 10 show the final gradation and snapshot of the numerical sample reconstructed in LMGC90 software, respectively.

\subsection{Burgers contact model and its calibration}

Asphalt mixture is a complex composite material showing a viscoelastic behavior, thus a viscoelastic contact model based on the Burgers model was adopted in the simulation. This model comprises a Maxwell model in series with a Kelvin-Voigt model (Fig. 11), the normal and tangential properties including stiffnesses and the viscosities for the Maxwell and the Kelvin-Voigt parts correspond to $K_{m i}, C_{m i}, K_{k i}$, and $C_{k i}$, respectively, where $\mathrm{i}$ stand for $\mathrm{n}, \mathrm{t}$ or $\mathrm{s}$.

For a viscoelastic material under cyclic loading, it is possible to determine the complex compliance from the resulting strain and stress as following:

$\left|D^{*}\right|=\sqrt{D^{\prime}(\omega)^{2}+D^{\prime \prime}(\omega)^{2}}$

$D^{\prime}(\omega)=\frac{1}{E_{m}}+\frac{E_{k}}{E_{k}^{2}+\omega^{2} \eta_{k}^{2}}$

$D^{\prime \prime}(\omega)=\frac{1}{\omega \eta_{m}}+\frac{\omega \eta_{k}}{E_{k}^{2}+\omega^{2} \eta_{k}^{2}}$

Where:

- $E_{m}, \eta_{m}, E_{k}$ and $\eta_{k}$ correspond to the macroscopic Maxwell and KelvinVoigt stiffnesses and viscosities respectively.
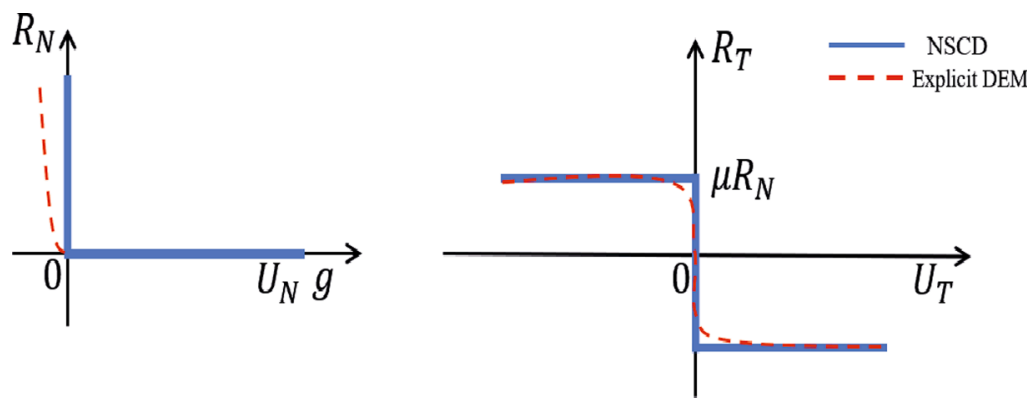

Fig. 8. Comparison between the two DEM modeling methods: Normal (left) and Tangential (right). 


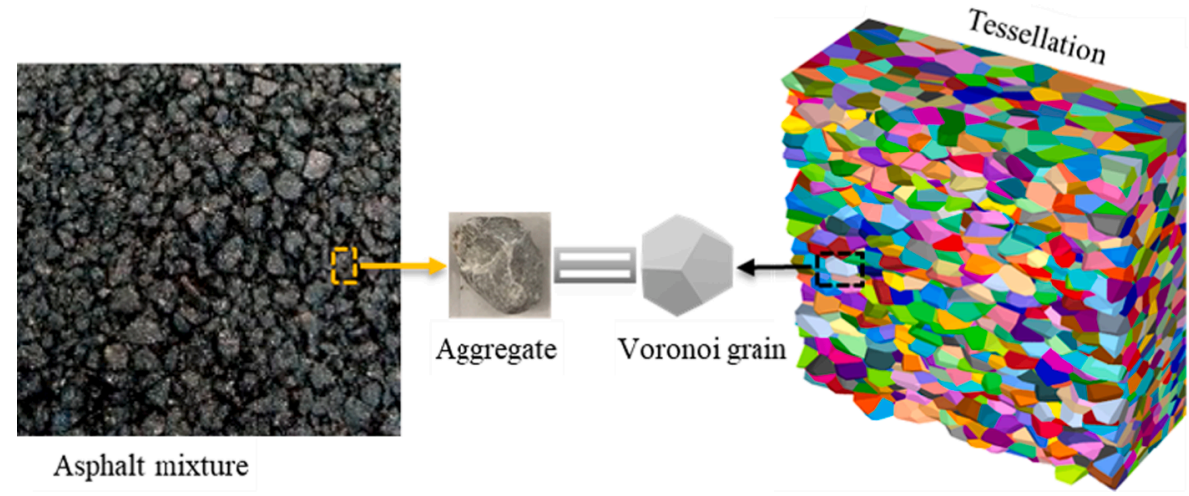

Fig. 9. Asphalt mixture and Voronoi tessellations.
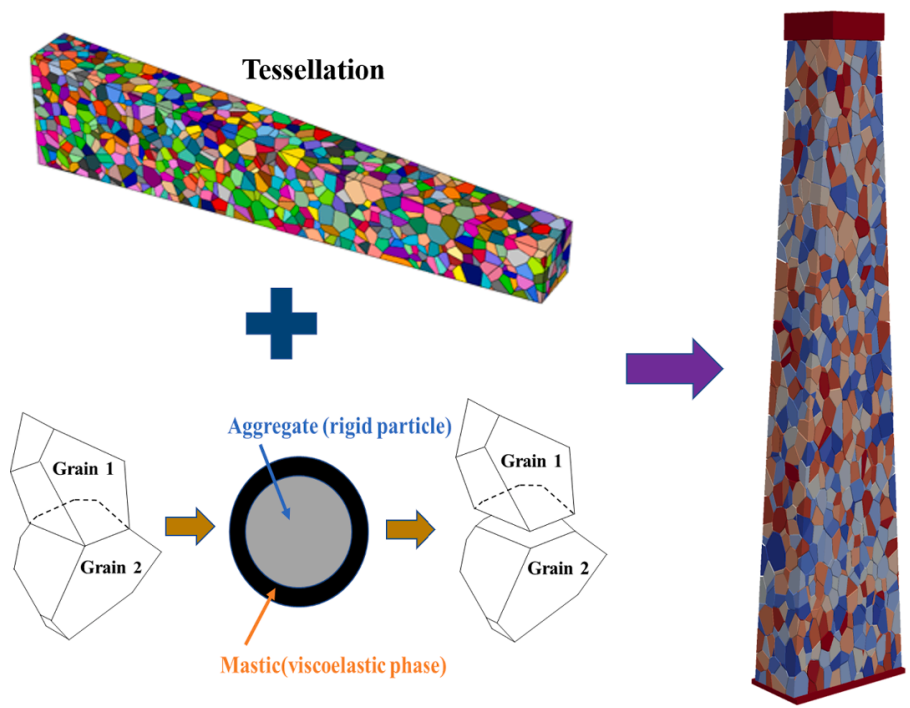

Viscoelastic behavior

Numerical sample

Fig. 10. Numerical modeling of test sample.

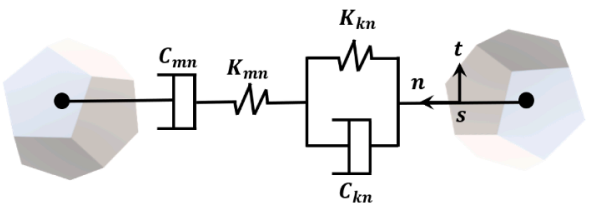

(a) Normal components

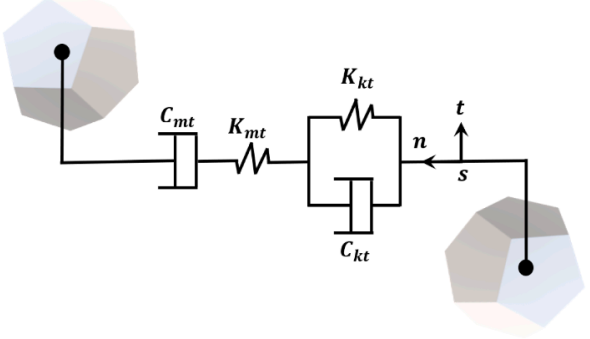

(b) Tangential components

Fig. 11. Burgers contact model between particles.
- $\left|D^{*}\right|$ is the complex compliance, $D^{\prime}$ and $D^{\prime \prime}$ are the real and the imaginary parts of the complex compliance, and $\omega$ is the pulsation.

The normal components of the microscopic parameters at the particle-scale can be assessed by:

$K_{m n}=A_{c} E_{m} / l_{0}$

$C_{m n}=A_{c} \eta_{m} / l_{0}$

$K_{k n}=A_{c} E_{k} / l_{0}$

$C_{k n}=A_{c} \eta_{k} / l_{0}$

Where:

- $A_{c}$ is the cross-section area of two particles in contact and $l_{0}$ is the initial distance between the particles.

On the other hand, the tangential components of the contact model for both tangential directions $t$ and $s$ can be computed from the above normal parameters, as:

$K_{m t}=K_{m s}=\frac{K_{m n}}{2(1+\nu)}$ 
$C_{m t}=C_{m s}=\frac{C_{m n}}{2(1+\nu)}$

$K_{k t}=K_{k s}=\frac{K_{k n}}{2(1+\nu)}$

$C_{k t}=C_{k s}=\frac{C_{k n}}{2(1+\nu)}$

Where:

- $\nu$ is the Poisson's ratio, this ratio was set to 0.35 [51].

The relations between velocities and contact forces for the Burgers model in CD method are plotted in Fig. 12. More details about the numerical implementation of this contact model in CD method can be found in [51].

The macroscopic model parameters allow determining the dynamic modulus as $\left|E^{*}\right|=\left|D^{*}\right|^{-1}$ and the phase angle by $\Phi=\arctan \left(D^{\prime \prime} / D^{\prime}\right)$. Using the established analytical expressions for $\left|E^{*}\right|$ and $\Phi$, the best fit of macroscopic parameters regarding experimental data can be estimated. The parameter calibration of Burgers model for each temperature was based on an optimization iterative procedure, as follows: (i) Performing the numerical simulation based on initial Burgers parameters under each loading frequency; (ii) Computing the numerical $\left|E^{*}\right|$ and $\Phi$ values from simulation results according to Eqs. 1 to 6; (iii) Correcting the analytical model (Eqs. 8 to 10) with the same parameters to fit the numerical values. After the calculation, a difference between the analytical and the numerical values could be observed. The correction step compensating the difference was processed by an optimization algorithm implemented in Python programming language based on the least-squares method; (iv) Using the corrected expressions above to fit the experimental $\left|E^{*}\right|$ and $\Phi$ values; (v) Evaluating the error between experimental and numerical $\left|E^{*}\right|$ and $\Phi$ values, the parameters fitted from this iteration step can be used to perform a new iteration step until the convergence criterion is achieved. Finally, after several iterations of model parameters adjustment, the model parameters were fitted optimally to the experimental data.

\section{Numerical analysis}

\subsection{Macro mechanical behavior}

Fig. 13 shows an example of the measured displacement and the corresponding force at the top plate for five loading cycles. As expected, both the force and displacement curves follow the sinusoidal wave. The phase angle between these two curves allows calculating $\psi$.

The master curve describing the loading time dependency of asphalt mixtures can be built according to the time-temperature superposition principle $[52,53]$. To build the corresponding master curve, a reference temperature $T_{\text {ref }}$ was chosen, then the translation of all isotherm values

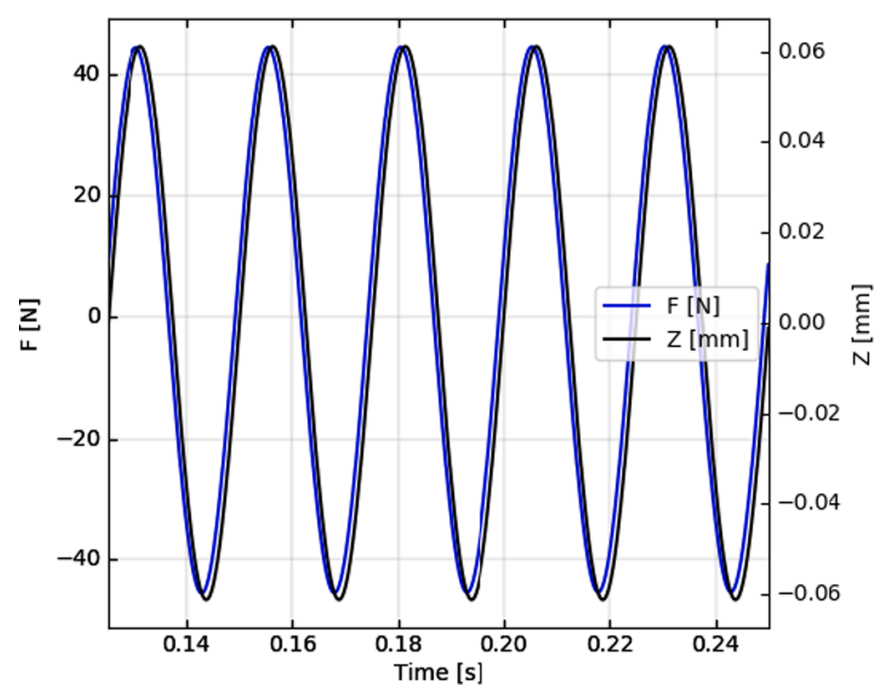

Fig. 13. Measured displacement $\mathrm{Z}$ and corresponding force $\mathrm{F}$ for $15^{\circ} \mathrm{C}$ and $40 \mathrm{~Hz}$.

was performed by calculating the reduced frequency as $f_{r}=a_{T} \times f$, where $f$ corresponds to each frequency value in each curve and $a_{T}$ is the shift factor. These values are obtained from the temperature fit using the Williams-Landel-Ferry (WLF) equation (Eq. (19) [54]):

$\log a_{T}=\frac{-C_{1}\left(T-T_{r e f}\right)}{C_{2}+\left(T-T_{r e f}\right)}$

Where:

- $C_{1}$ and $C_{2}$ are two constants, and $\mathrm{T}$ is the test temperature.

Fig. 14 shows the experimental and numerical results for the master curve with $C_{1}=28$ and $C_{2}=206.8^{\circ} \mathrm{C}$, at the reference temperature of $15^{\circ} \mathrm{C}$, which is a referenced value in French pavement design [40].

The numerical values from simulations are in good agreement with experimental values for $\left|E^{*}\right|$ and $\Phi$ for all the tested frequencies, despite some fluctuations around the average values. The best fitting parameters for the Burgers contact model are listed in Table 3.

\subsection{Micro mechanical behavior}

\subsubsection{Contact force distribution}

After the calibration, the previous stabilized specimens were submitted to dynamic loading. Fig. 15 illustrates the macro- and micromechanical behavior of an asphalt specimen in one loading cycle. At the macro-mechanical scale, repeating compression and tension states in the specimen lead to fatigue cracks inside the sample. On the other hand, at the contact-scale, there are two main types of force between particles:
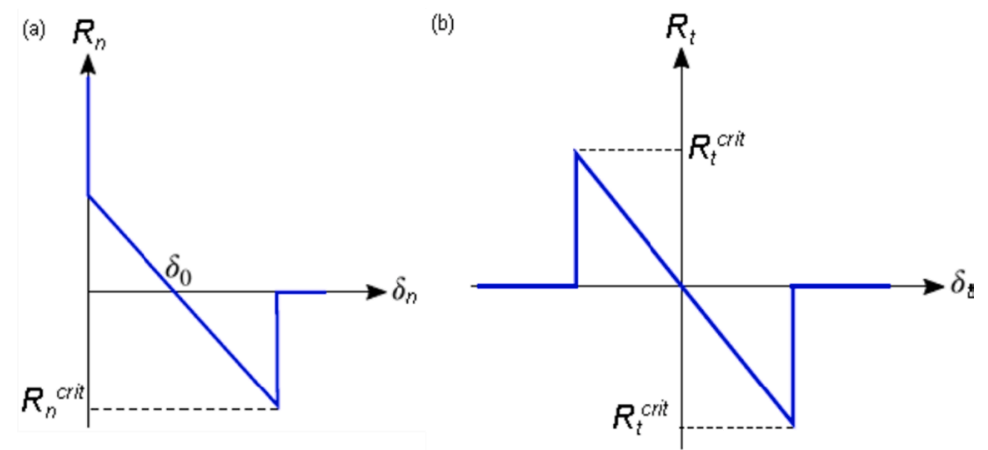

Fig. 12. Relations between velocities and contact forces: (a) Normal components (b) Tangential components [51]. 


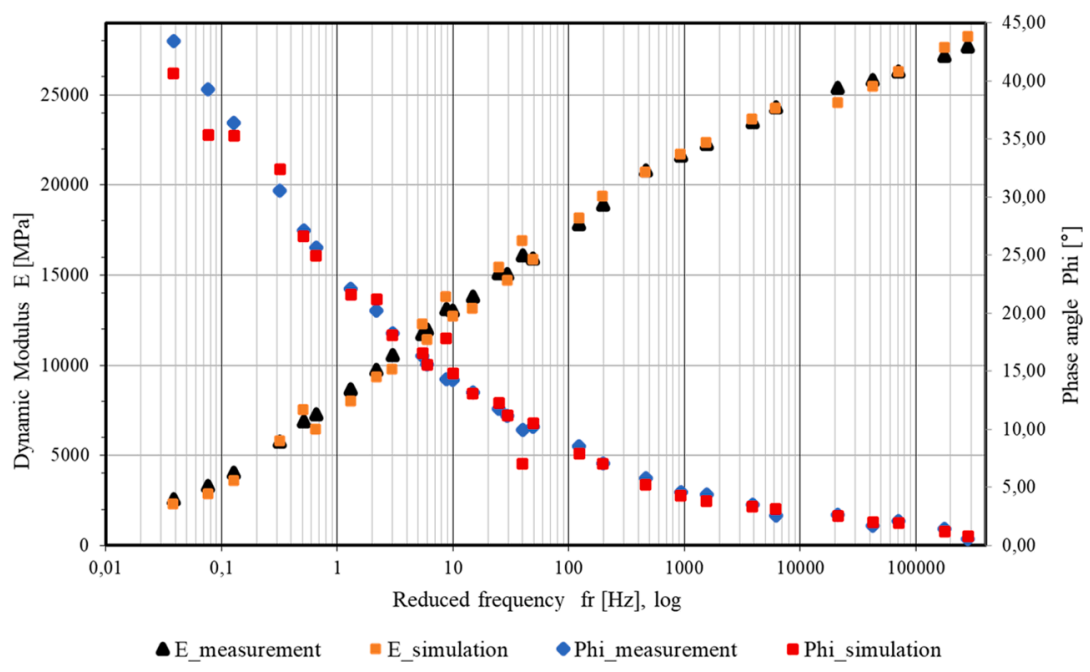

Fig. 14. Experimental and numerical master curve at $T_{r e f}=15^{\circ} \mathrm{C}$.

Table 3

Burgers model parameters used in numerical simulations.

\begin{tabular}{lllll}
\hline $\mathrm{T}\left({ }^{\circ} \mathrm{C}\right)$ & $E_{m}(\mathrm{~Pa})$ & $\eta_{m}$ (Pa.s) & $E_{k}(\mathrm{~Pa})$ & $\eta_{k}(\mathrm{~Pa} . \mathrm{s})$ \\
\hline-10 & $1.34 \times 10^{8}$ & $1.79 \times 10^{8}$ & $2.55 \times 10^{9}$ & $3.26 \times 10^{7}$ \\
0 & $1.18 \times 10^{8}$ & $6.86 \times 10^{7}$ & $9.36 \times 10^{8}$ & $1.03 \times 10^{7}$ \\
10 & $1.02 \times 10^{8}$ & $1.93 \times 10^{7}$ & $2.80 \times 10^{8}$ & $3.05 \times 10^{6}$ \\
15 & $9.48 \times 10^{7}$ & $1.02 \times 10^{7}$ & $1.70 \times 10^{8}$ & $1.79 \times 10^{6}$ \\
20 & $8.64 \times 10^{8}$ & $4.81 \times 10^{6}$ & $1.02 \times 10^{8}$ & $1.05 \times 10^{6}$ \\
30 & $7.18 \times 10^{7}$ & $8.84 \times 10^{5}$ & $3.39 \times 10^{7}$ & $2.80 \times 10^{5}$ \\
\hline
\end{tabular}

the tangential force inducing shearing in the interfacial plane of contact and the normal force lying perpendicular to this plane. Both forces contribute to the fatigue damage of asphalt mastic between particles, playing a major role in contact breakage and particle movement, finally initiating and propagating macro-cracks inside the mixture.

Figs. 16 and 17 show the normal stress and tangential stress distribution in one loading cycle, respectively. Both normal force value and tangential force value converge at zero at $\mathrm{T} 4$, which is consistent with the end loading condition. For the other loading points from T0 to T3, the distribution of the normal force values follows a normal distribution centered at zero, while the tangential force values develop an asymmetric distribution on one side of the x-axis. These distributions imply that the normal forces are symmetric around zero (tension-compression) during the loading cycle, while tangential forces change the direction and intensity as the loading condition changes. The horizontal displacement achieves a maximum value when both normal and tangential force are close to their maximums (because of the phase lag), according to $\mathrm{T} 1$ and $\mathrm{T} 3$.

\subsubsection{Particle velocity distribution and contact force networks}

The numerical sample follows the loading condition and responding particle movement behaves accordingly. Fig. 18 depicts different stages during one loading cycle in the numerical test. The particle velocity achieves the maximum value at $\mathrm{T} 1$ and $\mathrm{T} 3$ when the displacement amplitude is the maximum, and it is close to zero at T4 due to the balance calculation at the end of the loading. For T0 to T3, the average particle velocity displays an increasing trend along with the specimen height, from a minimum value for particles lying on the bottom plate up to a maximum value for particles bonded to the top loading plate.

In particle-based methods, the contact force network reflects the transmission of external loads in granular materials. Previous observations show that $\mathrm{T} 1$ and $\mathrm{T} 3$ are two characteristic points for the loaded specimen; therefore it is rewarding to visualize the contact force networks at these two points. Strong and weak contacts are always coexisting in a contact network, which forms the corresponding networks of strong contact forces, i.e. forces above the mean normal force $\left.\left(f_{n}\right\rangle\left\langle f_{n}\right\rangle\right)$, and weak contact forces $\left(f_{n}<\left\langle f_{n}\right\rangle\right)$ [28]. Fig. 19 shows the strong and weak force distribution at T1 and T3. Both strong (red) and weak forces (blue) change direction following the change in direction of the tangential force. This is consistent with the inference above.
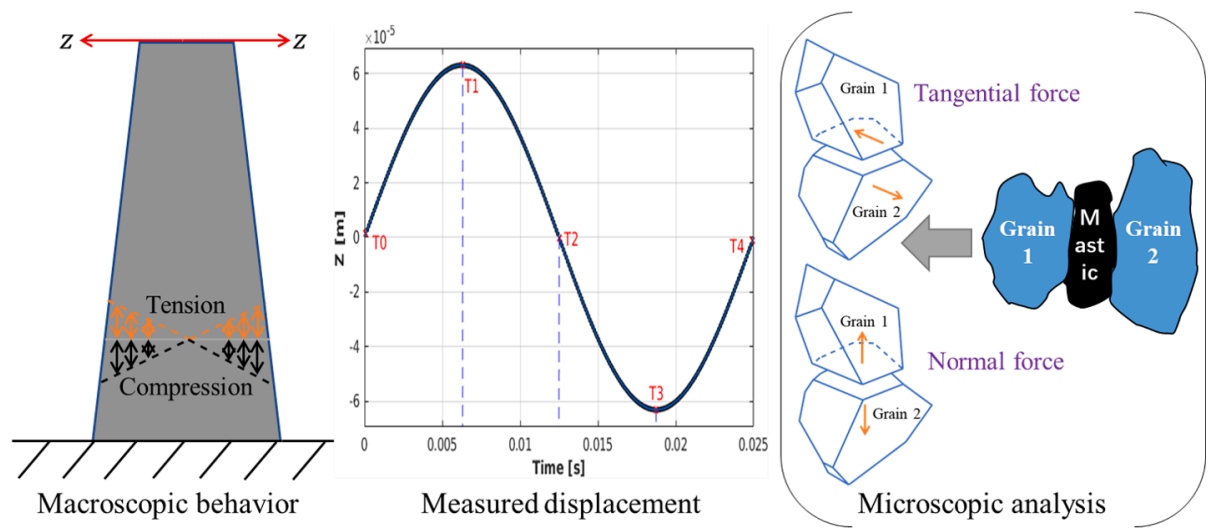

Fig. 15. Macro and micro behavior of asphalt specimen in one loading cycle. 


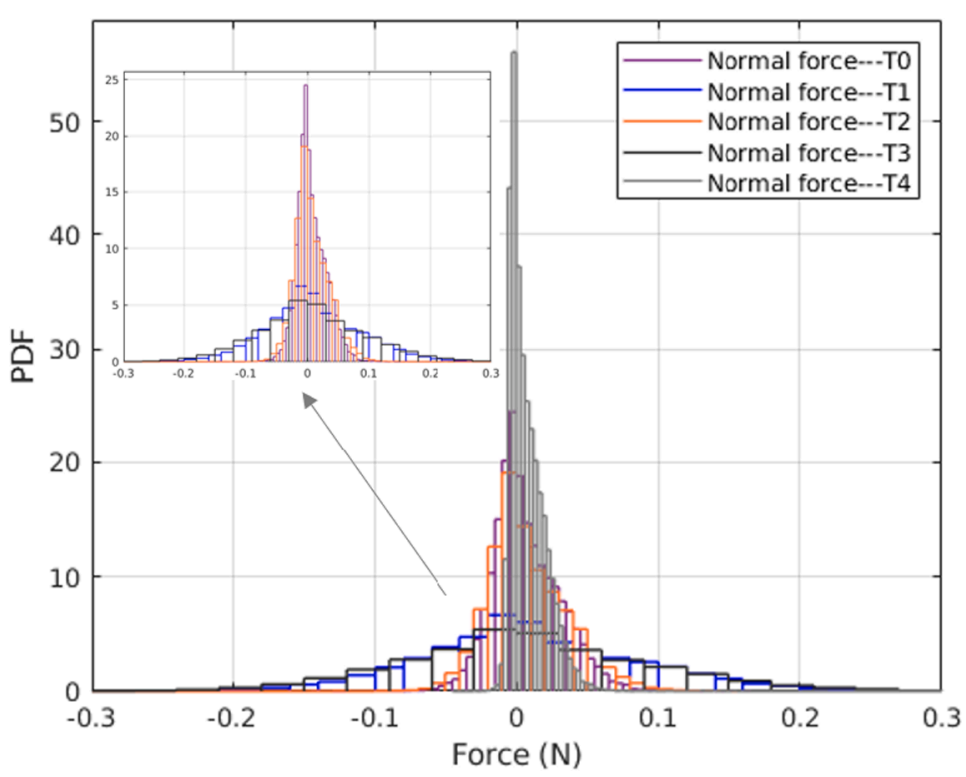

Fig. 16. Normal force probability distribution at $15^{\circ} \mathrm{C}, 40 \mathrm{~Hz}$.

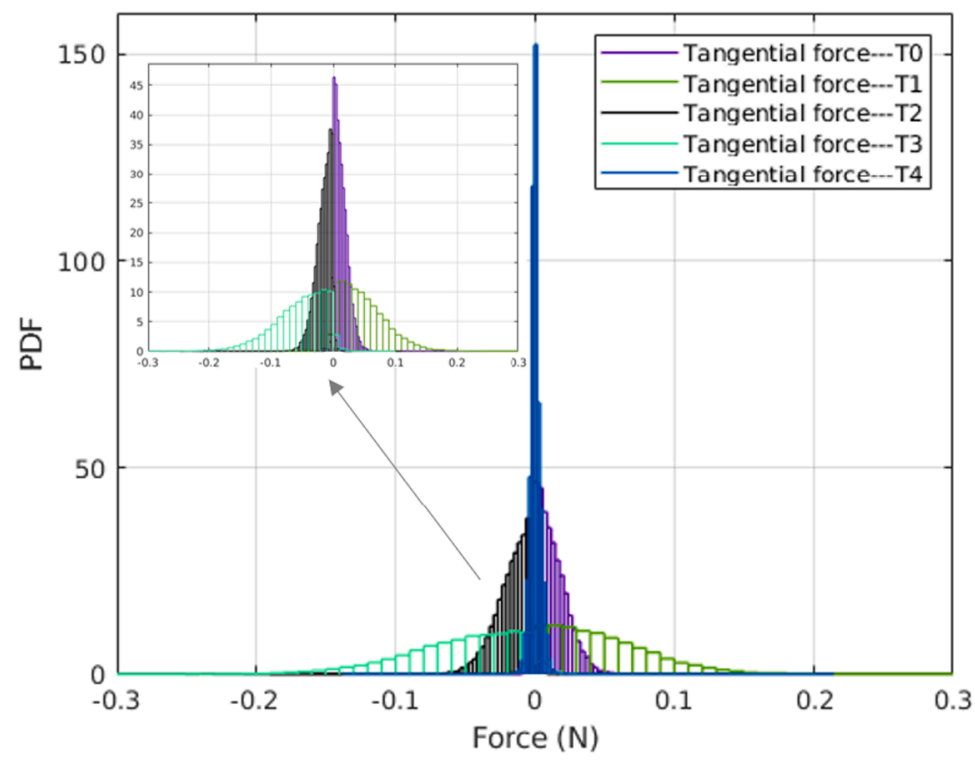

Fig. 17. Tangential force probability distribution at $15{ }^{\circ} \mathrm{C}, 40 \mathrm{~Hz}$.

\subsubsection{Normal and tangential force analysis}

As previously evoked, both normal and tangential forces between particles can be considered for the set up of a damage contact model. In this part, we examine the changing trend of the tangential and normal forces for different loading frequencies and temperatures. From the above analysis, we choose T1 and T3 as the critical time to process force analysis. Fig. 20 shows the changing trend of the two types of force at 15 ${ }^{\circ} \mathrm{C}$ for different frequencies. The tangential force reaches positive values at $\mathrm{T} 1$, where the measured displacement is maximum following the Xdirection. After a half loading cycle, the tangential force achieves negative values at $\mathrm{T} 3$, where the displacement reaches a maximum value in the opposite direction. The higher the frequency, the larger the magnitude of tangential force at $\mathrm{T} 1$ and $\mathrm{T} 3$ will be. The normal force has a wider distribution range than the tangential force and distributes symmetrically along the $\mathrm{Y}$-axis $(\mathrm{x}=0)$. Besides the range increases as the frequency increases, which indicates that the magnitude of the normal force shows the same trend as the tangential force.

Fig. 21 shows the changing trend of the tangential force and normal force at $40 \mathrm{~Hz}$ loading frequency for different temperatures. It can be seen that the magnitude of the tangential force decreases when the temperature increases. Similarly, the magnitude of normal force behaves in the same downtrend as the tangential force as the temperature increases. It also illustrates that the asphalt mixture is sensitive to temperature and the loading frequency, due to its viscoelastic nature.

At the macroscopic scale, asphalt materials have a stiffer behavior at higher frequencies or lower temperatures. During a performance test of asphalt mixtures, the specimen exchanges compression and tension in loading cycles. The specimen is subjected to a higher stress level at higher frequencies or lower temperatures, which induces earlier damage initiation.From the microscopic analysis at the contact scale above, we can conclude that the specimen withstanding lower frequency or higher temperature tends to exhibit better resisting performance to adhesive failure because of lower tangential force and lower normal forces between particles.

Particle forces analysis above reveals the micro-mechanism of mixture failure initiation at the contact scale. By using the presented 


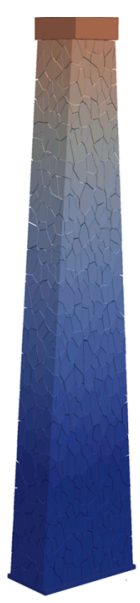

T0

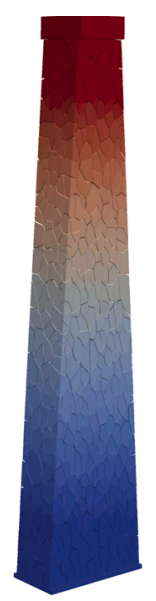

T1

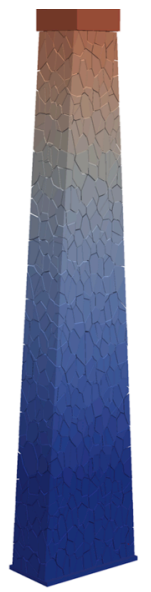

T2

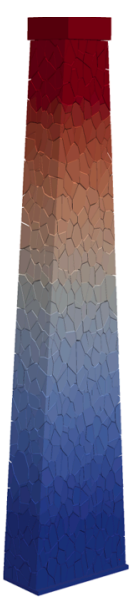

T3

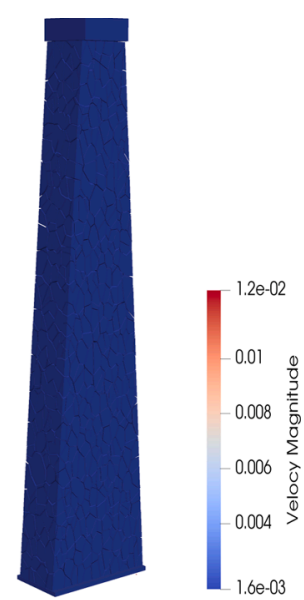

T4

Fig. 18. Average velocity of particles for $15^{\circ} \mathrm{C}$ and $40 \mathrm{~Hz}$.

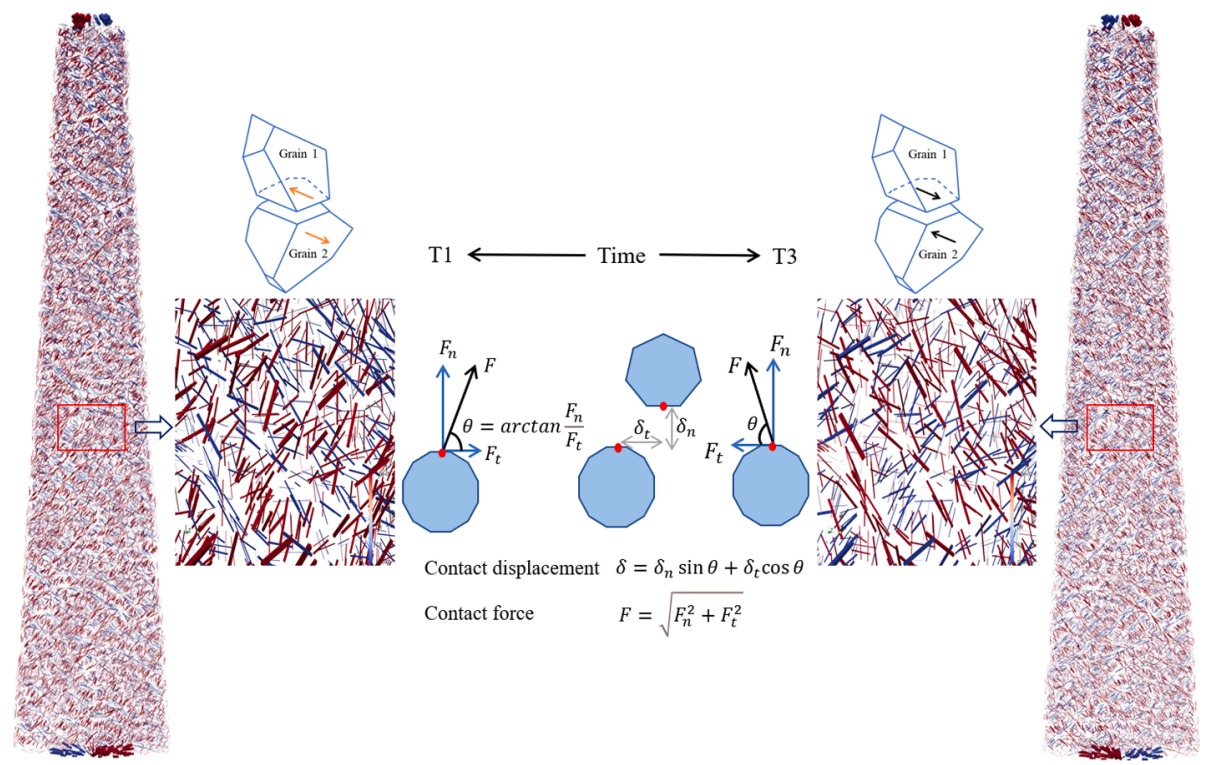

Fig. 19. Strong and weak contact force networks for $15{ }^{\circ} \mathrm{C}$ and $40 \mathrm{~Hz}$.

numerical method, some earlier failure patterns of asphalt mixtures could be envisaged. The findings here are also encouraging for further prediction of damage evolution in future studies. Long-term performance tests such as laboratory fatigue tests are always time-consuming and expensive. Due to the limitation of computation capacity, it is still challenging to reproduce long-term performance tests with larger numbers of loading cycles in numerical tests. The proposed numerical modeling method might be used to study mixture damage evolution if a damage model of bonds between particles with the cycle number is added in the numerical simulation process.

\section{Conclusion}

In this study, combining laser scanning and image processing, morphology features of aggregates were collected and analyzed statistically. Aggregate flatness ratio, elongation ratio, and Sphericity value all obey normal distribution. The average aspect ratio $(L: I: S)$ for aggregates and the mean and standard deviation of the Sphericity value were obtained as input particle shape parameters in the numerical simulation.

The contact dynamics method is introduced to model asphalt mixtures because of its superior capability in polyhedrons simulation. Asphalt mixture specimens were generated by a tessellation method based on the particle morphology statistics. The modeling method incorporate the aggregate morphology and size distribution in the numerical simulation. It also reduces the computational time significantly during the preparation stages for polyhedral samples, such as deposition by gravity and compaction.

Experimental and numerical 2 PB tests under different temperatures and frequencies were carried out to validate the model reliability. The dynamic modulus and the phase angle were calculated to analyze the viscoelastic behavior. The corresponding numerical master curves were in good agreement with experimental data. Through the microscopic analysis, both the tangential force and the normal force between particles have an apparent increasing trend with increasing loading frequency and decreasing temperature. Comparing the trend of the tangential force and normal force between the particles during cyclic loading, the mechanism of damage initiation for viscoelastic materials could be revealed at the particle-scale.

In conclusion, the modeling method presented in this study provides a basis for the high-precision simulation of asphalt mixtures composing of polyhedral particles. It can be seen as an appealing alternative to the 


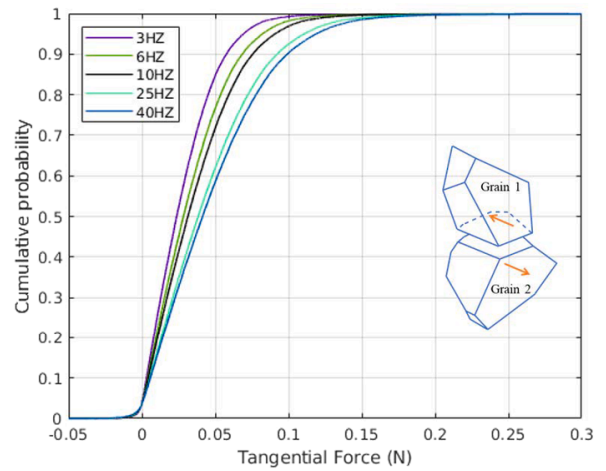

(a) Tangential force at $\mathrm{T} 1$

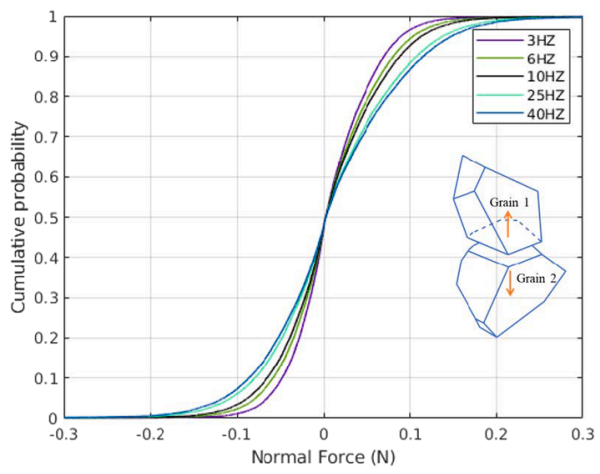

(c) Normal force at T1

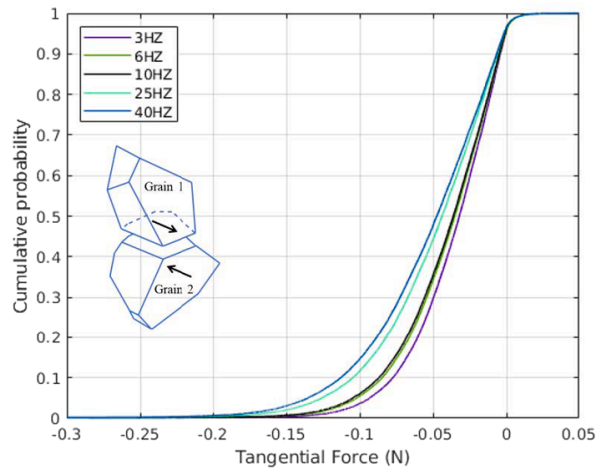

(b) Tangential force at T3

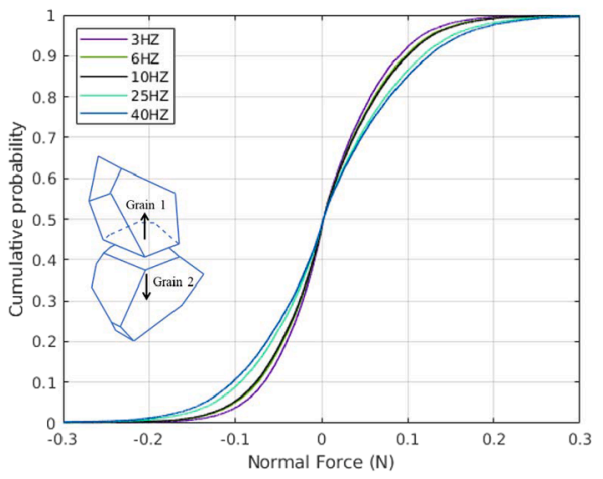

(d) Normal force at T3

Fig. 20. Cumulative distribution for tangential force and normal force at $\mathrm{T} 1, \mathrm{~T} 3,15{ }^{\circ} \mathrm{C}$.

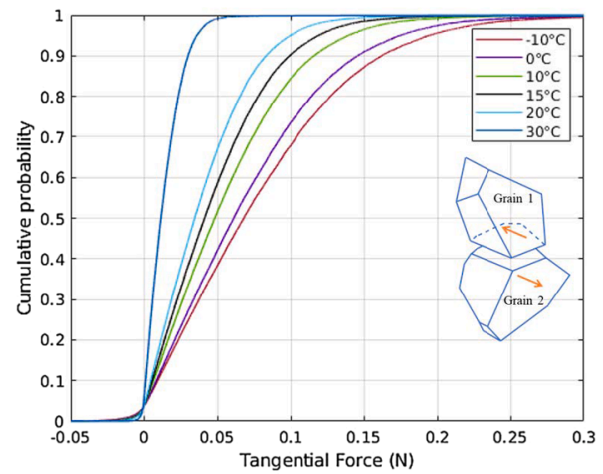

(a) Tangential force at $\mathrm{T} 1$

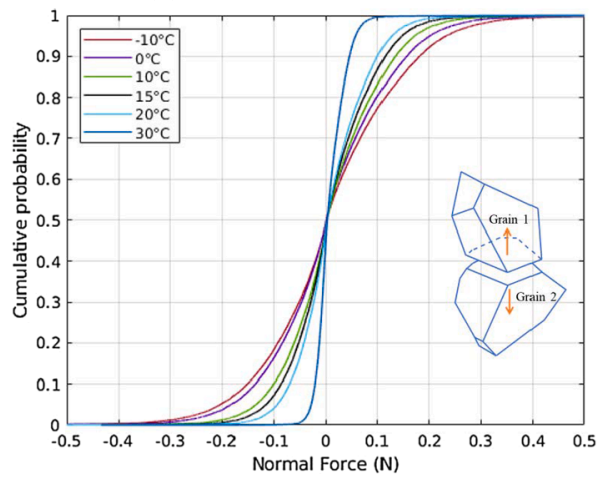

(c) Normal force at T1

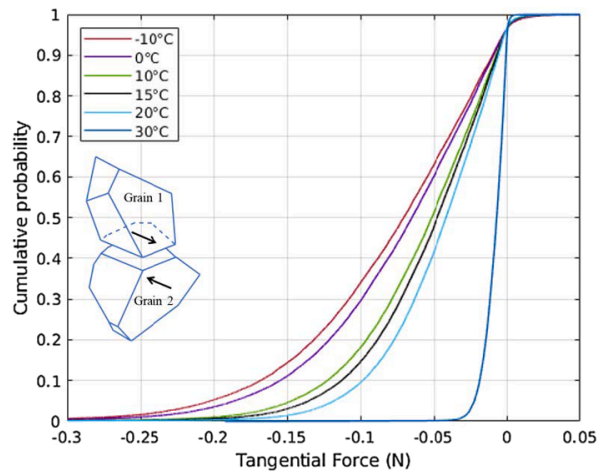

(b) Tangential force at T3

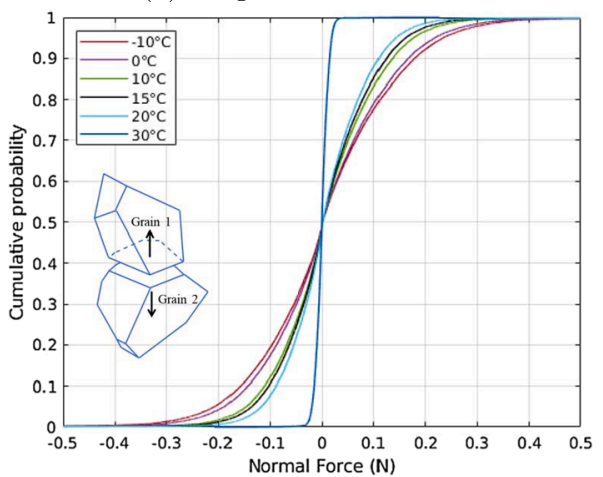

(d) Normal force at T3

Fig. 21. Cumulative distribution for tangential force and normal force at T1, T3, $40 \mathrm{~Hz}$. 
classic procedures. Future works will focus on the effect of different particle size and morphology distribution and rheological properties of asphalt mixtures.

\section{CRediT authorship contribution statement}

Haitao Ge: Conceptualization, Software, Investigation, Writing original draft, Visualization. Juan Carlos Quezada: Conceptualization, Software, Writing - review \& editing. Vincent Le Houerou: Conceptualization, Writing - review \& editing. Cyrille Chazallon: Conceptualization, Writing - review \& editing, Funding acquisition.

\section{Declaration of Competing Interest}

The authors declare that they have no known competing financial interests or personal relationships that could have appeared to influence the work reported in this paper.

\section{Acknowledgements}

The work presented in this paper was financially supported by the French National Research Agency (ANR-SolDuGri project ANR-14CE22-0019). The first author gratefully acknowledge financial support from China Scholarship Council (No. 201806560055).

\section{References}

[1] M.H. Sadd, Q. Dai, V. Parameswaran, A. Shukla, Microstructural simulation of asphalt materials: Modeling and experimental studies, Journal of Materials in Civil Engineering 16 (2) (2004) 107-115.

[2] H. Yu, S. Shen, A micromechanical based three-dimensional dem approach to characterize the complex modulus of asphalt mixtures, Construction and Building Materials 38 (2013) 1089-1096.

[3] W. Cai, G. McDowell, G. Airey, Discrete element visco-elastic modelling of a realistic graded asphalt mixture, Soils and Foundations 54 (1) (2014) 12-22.

[4] F. Gong, Y. Liu, X. Zhou, Z. You, Lab assessment and discrete element modeling of asphalt mixture during compaction with elongated and flat coarse aggregates, Construction and Building Materials 182 (2018) 573-579.

[5] H.M. Zelelew, A.T. Papagiannakis, Micromechanical modeling of asphalt concrete uniaxial creep using the discrete element method, Road Materials and Pavement Design 11 (3) (2010) 613-632.

[6] M. Moaveni, E. Mahmoud, E.M. Ortiz, E. Tutumluer, S. Beshears, Use of advanced aggregate imaging systems to evaluate aggregate resistance to breakage, abrasion, and polishing, Transportation Research Record 2401 (1) (2014) 1-10.

[7] T. Bennert, L.A. Cooley Jr, C. Ericson, Z. Zavery, Coarse aggregate angularity and its relationship to permanent deformation of gravel-aggregate hot-mix asphalt in New York State, Transportation Research Record 2207 (1) (2011) 25-33.

[8] J.-S. Chen, W. Hsieh, M.-C. Liao, Effect of coarse aggregate shape on engineering properties stone mastic asphalt applied to airport pavements, International Journal of Pavement Research and Technology 6 (5) (2013) 595.

[9] L.T. Souza, Y.-R. Kim, F.V. Souza, L.S. Castro, Experimental testing and finiteelement modeling to evaluate the effects of aggregate angularity on bituminous mixture performance, Journal of Materials in Civil Engineering 24 (3) (2012) 249-258.

[10] D. Castillo, S. Caro, M. Darabi, E. Masad, Influence of aggregate morphology on the mechanical performance of asphalt mixtures, Road Materials and Pavement Design 19 (4) (2018) 972-991.

[11] Z. You, W.G. Buttlar, Discrete element modeling to predict the modulus of asphalt concrete mixtures, Journal of Materials in Civil Engineering 16 (2) (2004) $140-146$.

[12] Z. You, W.G. Buttlar, Application of discrete element modeling techniques to predict the complex modulus of asphalt-aggregate hollow cylinders subjected to internal pressure, Transportation Research Record 1929 (1) (2005) 218-226.

[13] M.J. Khattak, A. Khattab, H.R. Rizvi, S. Das, M.R. Bhuyan, Imaged-based discrete element modeling of hot mix asphalt mixtures, Materials and Structures 48 (8) (2015) 2417-2430.

[14] Z. You, S. Adhikari, Q. Dai, Three-dimensional discrete element models for asphalt mixtures, Journal of Engineering Mechanics 134 (12) (2008) 1053-1063.

[15] Y. Fu, L. Wang, C. Zhou, 3d clustering dem simulation and non-invasive experimental verification of shear localisation in irregular particle assemblies, International Journal of Pavement Engineering 11 (5) (2010) 355-365.

[16] S. Adhikari, Z. You, M.E. Kutay, Prediction of dynamic modulus of asphalt concrete using two-dimensional and three-dimensional discrete element modeling approach, GeoCongress 2008: Characterization, Monitoring, and Modeling of GeoSystems (2008) 1020-1027.

[17] J. Wimmer, B. Stier, J.-W. Simon, S. Reese, Computational homogenisation from a 3d finite element model of asphalt concrete-linear elastic computations, Finite Elements in Analysis and Design 110 (2016) 43-57.
[18] Y. Liu, Z. You, Visualization and simulation of asphalt concrete with randomly generated three-dimensional models, Journal of Computing in Civil Engineering 23 (6) (2009) 340-347.

[19] D. Zhang, X. Huang, Y. Zhao, Algorithms for generating three-dimensional aggregates and asphalt mixture samples by the discrete-element method, Journal of Computing in Civil Engineering 27 (2) (2013) 111-117.

[20] T. Ma, D. Zhang, Y. Zhang, S. Wang, X. Huang, Simulation of wheel tracking test for asphalt mixture using discrete element modelling, Road Materials and Pavement Design 19 (2) (2018) 367-384.

[21] C. Zhou, X. Liu, P. Apostolidis, A.T. Scarpas, L. He, Induction heating-assisted compaction in Porous Asphalt Pavements: A computational study, Applied Sciences 8 (11) (2018) 2308.

[22] B. Xue, J. Pei, B. Zhou, J. Zhang, R. Li, F. Guo, Using random heterogeneous dem model to simulate the scb fracture behavior of asphalt concrete, Construction and Building Materials 236 (2020), 117580.

[23] X. Ding, T. Ma, W. Gao, Morphological characterization and mechanical analysis for coarse aggregate skeleton of asphalt mixture based on discrete-element modeling, Construction and Building Materials 154 (2017) 1048-1061.

[24] Y. Liu, X. Zhou, Z. You, S. Yao, F. Gong, H. Wang, Discrete element modeling of realistic particle shapes in stone-based mixtures through matlab-based imaging process, Construction and Building Materials 143 (2017) 169-178.

[25] X. Zhou, Y. Liu, Z. You, Heavy impact compaction modeling and analysis on unbound paving mixtures, in: International Conference on Discrete Element Methods, Springer, 2016, pp. 437-444.

[26] N. Das. Modeling three-dimensional shape of sand grains using discreteelement method, University of South Florida, 2007.

[27] F. Alonso-Marroquin, H.J. Herrmann, Calculation of the incremental stress-strain relation of a polygonal packing, Physical Review E 66 (2002), 021301, https://doi. org/10.1103/PhysRevE.66.021301. URL: https://link.aps.org/doi/10.1103/ PhysRevE.66.021301.

[28] E. Azéma, F. Radjai, G. Saussine, Quasistatic rheology, force transmission and fabric properties of a packing of irregular polyhedral particles, Mechanics of Materials 41 (6) (2009) 729-741.

[29] T. Al-Rousan, E. Masad, E. Tutumluer, T. Pan, Evaluation of image analysis techniques for quantifying aggregate shape characteristics, Construction and Building Materials 21 (5) (2007) 978-990.

[30] T. Pan, E. Tutumluer, S.H. Carpenter, Effect of coarse aggregate morphology on the resilient modulus of hot-mix asphalt, Transportation Research Record 1929 (1) (2005) 1-9.

[31] E. Tutumluer, T. Pan, Aggregate morphology affecting strength and permanent deformation behavior of unbound aggregate materials, Journal of Materials in Civil Engineering 20 (9) (2008) 617-627.

[32] I.S. Bessa, V.T.C. Branco, J.B. Soares, J.A.N. Neto, Aggregate shape properties and their influence on the behavior of hot-mix asphalt, Journal of Materials in Civil Engineering 27 (7) (2015) 04014212.

[33] Y. Zhang, T. Ma, X. Huang, Y. Zhao, P. Hu, Algorithms for generating air-void structures of idealized asphalt mixture based on three-dimensional discreteelement method, Journal of Transportation Engineering, Part B: Pavements 144 (2) (2018) 04018023.

[34] W. Jia, V. Markine, Y. Guo, G. Jing, Experimental and numerical investigations on the shear behaviour of recycled railway ballast, Construction and Building Materials 217 (2019) 310-320.

[35] Y. Sun, B. Indraratna, S. Nimbalkar, Three-dimensional characterisation of particle size and shape for ballast, Géotechnique Letters 4 (3) (2014) 197-202.

[36] H. Ge, A. Sha, Z. Han, X. Xiong, Three-dimensional characterization of morphology and abrasion decay laws for coarse aggregates, Construction and Building Materials 188 (2018) 58-67.

[37] N.P. 18-545:2011, Granulats - Éléments de définition, conformité et codification (in french). (2011).

[38] H. Wadell, Volume, shape, and roundness of quartz particles, The Journal of Geology 43 (3) (1935) 250-280.

[39] P. Liu, J. Hu, D. Wang, M. Oeser, S. Alber, W. Ressel, G.C. Falla, Modelling and evaluation of aggregate morphology on asphalt compression behavior, Construction and Building Materials 133 (2017) 196-208.

[40] C. EN, et al., Bituminous mixtures-test methods for hot mix asphalt-part 26: Stiffness (2012).

[41] M. Pérez-Martinez, Durabilité des enrobés tièdes intégrant des recyclés, Ph.D. thesis, Ecole centrale de Nantes, 2017.

[42] P.A. CUNDALL, A computer model for simulating progressive, large-scale movement in blocky rock system, Proceedings of the International Symposium on Rock Mechanics, 1971. URL: https://ci.nii.ac.jp/naid/10018723276/en/.

[43] P.A. Cundall, O.D. Strack, A discrete numerical model for granular assemblies, Geotechnique 29 (1) (1979) 47-65.

[44] J.J. Moreau, Unilateral contact and dry friction in finite freedom dynamics, in: Nonsmooth Mechanics and Applications, Springer, 1988, pp. 1-82.

[45] M. Jean, The non-smooth contact dynamics method, Computer Methods in Applied Mechanics and Engineering 177 (3-4) (1999) 235-257.

[46] F. Dubois, V. Acary, M. Jean, The contact dynamics method: A nonsmooth story, Comptes Rendus Mécanique 346 (3) (2018) 247-262.

[47] F. Radjai, V. Richefeu, Contact dynamics as a nonsmooth discrete element method, Mechanics of Materials 41 (6) (2009) 715-728.

[48] L. Gaillard, J.C. Quezada, C. Chazallon, P. Hornych, Resilient modulus prediction of rap using the contact dynamics method, Transportation Geotechnics 24 (2020), 100371. 
[49] R. Quey, P. Dawson, F. Barbe, Large-scale 3d random polycrystals for the finite element method: Generation, meshing and remeshing, Computer Methods in Applied Mechanics and Engineering 200 (17-20) (2011) 1729-1745.

[50] R. Quey, L. Renversade, Optimal polyhedral description of 3d polycrystals: method and application to statistical and synchrotron x-ray diffraction data, Computer Methods in Applied Mechanics and Engineering 330 (2018) 308-333.

[51] J.C. Quezada, C. Chazallon, Complex modulus modeling of asphalt concrete mixes using the non-smooth contact dynamics method, Computers and Geotechnics 117 (2020), 103255
[52] J.S. Daniel, A. Lachance, Mechanistic and volumetric properties of asphalt mixtures with recycled asphalt pavement, Transportation Research Record 1929 (1) (2005) 28-36.

[53] Y.R. Kim, S.J. Lee, Y. Seo, O. El-Haggan, A mechanistic approach to determine price reduction factors for density-deficient asphalt pavements, in: Airfield and Highway Pavement: Meeting Today's Challenges with Emerging Technologies, 2006, pp. 1018-1029.

[54] M.L. Williams, R.F. Landel, J.D. Ferry, The temperature dependence of relaxation mechanisms in amorphous polymers and other glass-forming liquids, Journal of the American Chemical society 77 (14) (1955) 3701-3707. 